\title{
Acute Pancreatitis and Kidney Injury Secondary to Paget- Schroetter Syndrome Following Percutaneous Mechanical Thrombectomy
}

\author{
Vincent Marcuccia, c ${ }^{\mathrm{a}}$, Sindi Diko ${ }^{\mathrm{b}}$, John Danks ${ }^{\mathrm{b}}$
}

\begin{abstract}
Paget-Schroetter syndrome (PSS) is an uncommon, though well-documented, cause of upper extremity deep vein thrombosis. Percutaneous mechanical thrombectomy and catheter-directed lysis of clot burden is considered an effective treatment for minimizing and removing deep vein thrombi, although several uncommon life-threatening sequelae may occur. Herein, we present a case of an otherwise healthy 19-year-old man who developed acute pancreatitis and kidney injury following successful treatment for PSS and discuss the possible inciting factors for pancreatic insult.
\end{abstract}

Keywords: Paget-Schroetter syndrome; Upper extremity deep venous thrombosis; Percutaneous mechanical thrombectomy; Catheterdirected thrombolysis; Acute pancreatitis; Acute kidney injury

\section{Introduction}

Effort-induced thrombosis, also known as Paget-Schroetter syndrome (PSS), is a result of dynamic, forceful upper extremity movements that can be associated with anatomic abnormalities including hypertrophied scalene musculature and extranumerary rib(s). The pathogenesis of PSS is hypothesized to be a result of vessel scarring, stenosis, and eventual thrombosis $[1,2]$. It has been widely accepted that the standard of treatment for PSS is catheter-directed thrombolytic therapy via transaxillary, infraclavicular, or paraclavicular approaches with combined anticoagulation [2, 3].

Percutaneous mechanical thrombectomy (PMT) offers a

Manuscript submitted June 14, 2021, accepted June 28, 2021

Published online September 30, 2021

aDepartment of Surgery, Jersey Shore University Medical Center, Neptune, NJ, USA

bDepartment of Surgery, St. Joseph's University Medical Center, Paterson, NJ, USA

${ }^{\mathrm{c} C o r r e s p o n d i n g ~ A u t h o r: ~ V i n c e n t ~ M a r c u c c i, ~ D e p a r t m e n t ~ o f ~ S u r g e r y, ~ J e r s e y ~}$ Shore University Medical Center, 1945 NJ Route 33, Neptune, NJ 07753, USA.Email: marcucv1@gmail.com

doi: https://doi.org/10.14740/jcs442 minimally invasive technique for removal of intravascular blood clot burden $[3,4]$. Rheolytic thrombectomy, as used in our case, is a procedure utilizing a pump driven catheter to deliver a highpressure propulsion of saline to the tip of the catheter, creating a vacuum that breaks the clot into fragments and suctions the fragments out of the vessel $[4,5]$. Lysis and clot removal with rheolytic PMT is commonly used to treat deep vein thrombosis due to the ease of use, high efficacy, and overall low morbidity [6]. However, PMT has been noted to have several significant adverse effects including fragmented embolization, arterial/venous perforation, fluid overload, cardiac arrhythmias, hemorrhagic events, acute kidney injury (AKI) due to myoglobinuria, and rare instances of acute pancreatitis (AP) [6-8].

This report details the potential source and management of AP when associated with intraoperative tissue plasminogen activator (tPA) and its breakdown products in a young patient undergoing mechanical thrombectomy for PSS without any predisposition to organ dysfunction.

\section{Case Report}

A healthy 19-year-old man with no past medical or significant family history presented with swelling and mild pain to his left upper extremity that began acutely after resistance training. The patient did not endorse previous episodes of excessive extremity swelling after exercising. An upper extremity duplex ultrasonography (US) was performed in the emergency room revealing left distal axillary and subclavian thrombosis, with subsequent computed tomography (CT) angiography confirming the US findings (Fig. 1).

A heparin drip was initiated and the decision was made to take the patient to the operating room (OR) for mechanical thrombectomy with intraoperative thrombolysis, intravascular ultrasound (IVUS), and placement of a lysis catheter in the left brachial, axillary, subclavian, and the brachiocephalic veins. Under ultrasound guidance, a micropuncture needle was used to puncture the left brachial vein and a wire was advanced under fluoroscopic guidance up the left brachial vein. X-ray fluoroscopy demonstrated nearly complete subclavian vein occlusion (Fig. 2). The angiojet rheolytic device was utilized to place 10 $\mathrm{mg}$ of alteplase over the thrombus and subsequently remove the fragmented blood clot. The patient tolerated the procedure without complication, followed by transfer to the pediatric intensive 


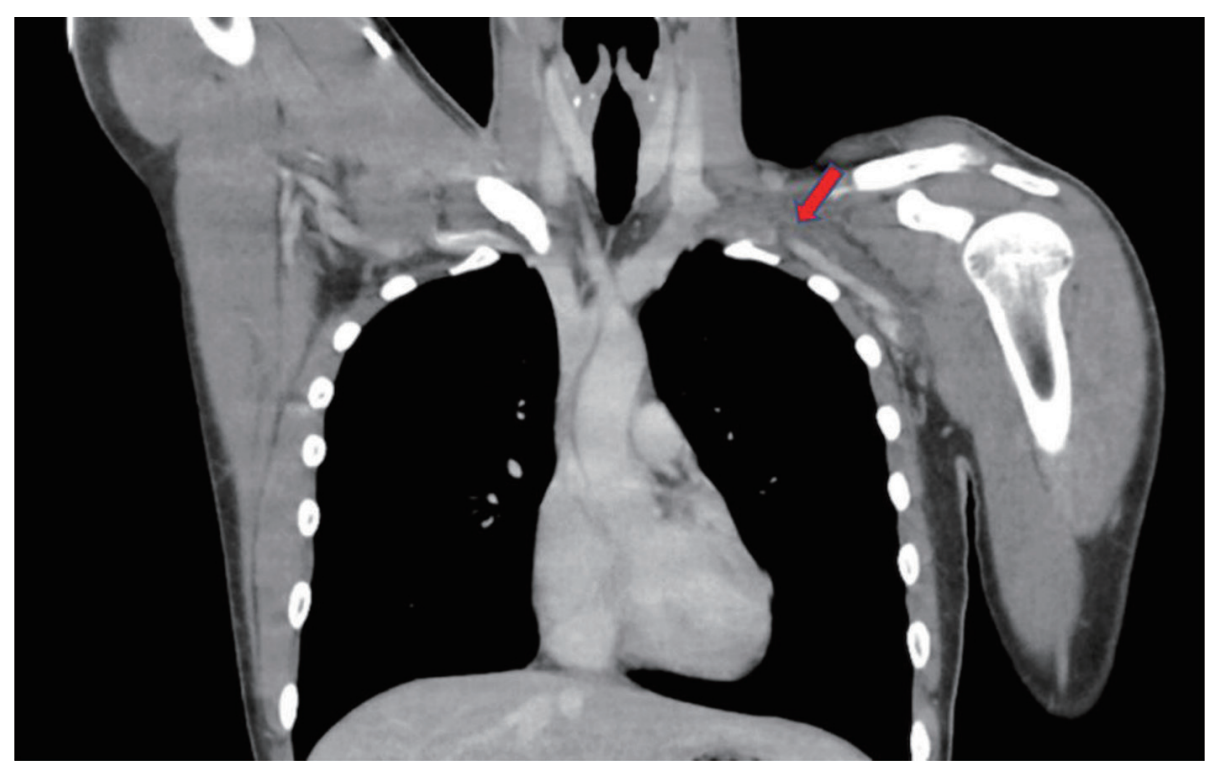

Figure 1. Computed tomography angiography signifying subclavian vein thrombosis. Red arrow points to occlusion.

care unit for ongoing catheter-directed thrombolysis. Approximately $4 \mathrm{~h}$ post-procedure, the patient began complaining of dark colored urine, severe epigastric abdominal pain refractory to medication, nausea, and emesis. Labs were drawn, returning an amylase and lipase level of $529 \mathrm{U} / \mathrm{L}$ (normal: 29 - $103 \mathrm{U} / \mathrm{L}$ ) and 3,171 U/L (normal: 11 - $82 \mathrm{U} / \mathrm{L}$ ), respectively, consistent with AP. Urinalysis showed evidence of hemolysis and a complete metabolic panel (CMP) consistent with AKI, as well.

Within $24 \mathrm{~h}$, the patient returned to the OR for repeat angiography and PMT. IVUS evaluated areas of venous compres- sion, demonstrating proximal subclavian venous narrowing and distal dilation at the level of the head of the left clavicle, raising suspicion for venous thoracic outlet syndrome (TOS). The patient remained hospitalized for four more days post intervention until he had resolution of his abdominal pain, AKI and upper extremity edema and pain. Hematology was consulted to pursue testing for potential hypercoagulable disorders. Blood work in our patient returned positive for a heterozygous factor $\mathrm{V}$ Leiden (FVL) mutation. The patient was advised that he would benefit from decompression of his TOS in order to avoid subsequent

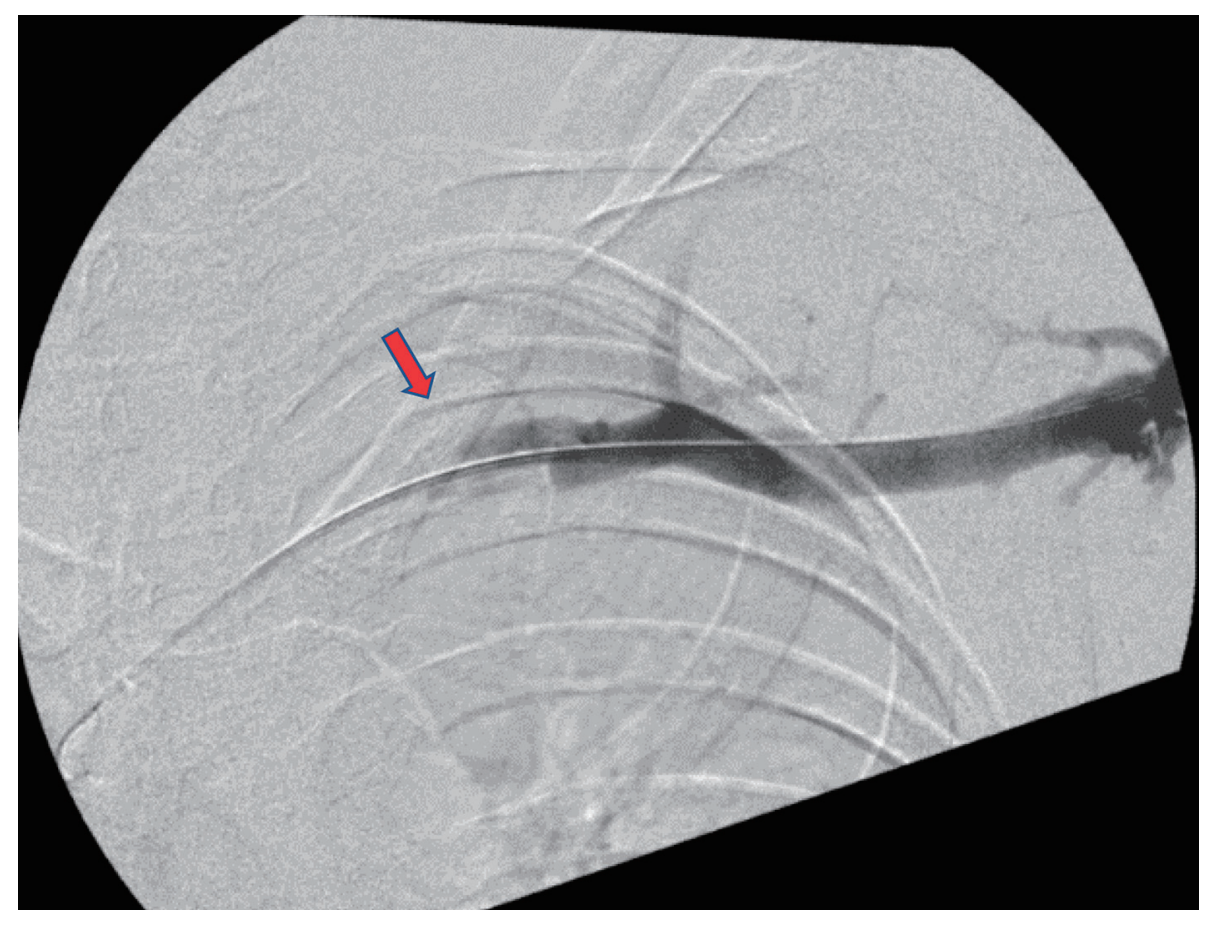

Figure 2. X-ray fluoroscopy displaying subclavian vein occlusion pre-thrombectomy. Red arrow points to occlusion. 
upper extremity thrombosis in the future, but the patient and family refused further surgery during this admission. He was discharged on rivaroxaban $15 \mathrm{mg}$ twice daily and, at a later date, the patient had successful decompressive surgery for TOS and recovered well from his procedures.

\section{Discussion}

PSS accounts for approximately $10-20 \%$ of all upper extremity deep vein thrombosis and is commonly found in young active individuals [1], much like our patient. Hypertrophied scalene and subclavius muscles from various physical activities (swimming and weight lifting) are believed to elicit subclavian venous endothelial damage and coagulation cascade activation, responsible for thrombus formation [9]. It has been regarded that many patients have also been diagnosed with underlying hypercoagulable disorders [1]. FVL mutation is an autosomal dominant mutation in clotting factor $\mathrm{V}$ that renders this protein resistant to protein $\mathrm{C}$ inactivation.

FVL mutation is strongly associated with vascular endothelial damage and inflammatory activation. In the event of thrombus formation, there is a possibility of intravascular hemolysis and microthrombi fragmentation with distal embolization [10]. In an 8-year comprehensive study at Johns Hopkins University studying the association between FVL mutation and PSS, 143 patients presented with subclavian venous thrombosis (SVT), with 55 patients being tested for hypercoagulable disorders and only two male patients testing positive for FVL mutation [11]. While FVL mutation can cause SVT, we hypothesize this is an unlikely explanation for our patient's AP.

The high velocity saline propulsion used in PMT to fracture and lyse thrombi frequently causes inadvertent erythrocyte damage and hemolysis $[6,12]$. A substantial hemolytic event is thought to be the driving force behind AKI in post-thrombectomy due to the release of hemoglobin and its breakdown products $[6,12]$, and may be an inciting factor in the occurrence of AP. Hemoglobin and hemoproteins are involved in neutrophil recruitment and degranulation, as well as the induction of coagulation cascade-induced vasoconstriction and inflammatory cytokine upregulation, such as platelet-activating factor, tumor necrosis factor, and $\alpha-2$ microglobulin, which can cause direct epithelial and obstructive damage within the pancreas [6-8, 12].

The possibility of AP as a sequela of acute or chronic medication administration, alcohol consumption, and biliary disease was considered, though, none of these pertained to our patient. The administration of propofol inducing AP was explored. In a systematic review of literature identifying 18 studies from 11 countries totaling 21 patients who suffered AP, intraoperative propofol was strongly associated with this post-operative finding [13]. This study concluded that a dose of $200 \mathrm{mg}$ of propofol given over approximately $2.33 \mathrm{~h}$ was found to be the average dose and time interval for patients who developed AP [13]. The timeframe from propofol administration and onset of pancreatitis occurred within $24 \mathrm{~h}$ in $28 \%$ of this patient population and within 1 week in $>50 \%$ [13]. This is similar to our case, in dosage $(200 \mathrm{mg})$ and intraoperative time (about $3 \mathrm{~h}$ ). Baseline renal function, total thrombectomy time, and intraoperative effluent should also be considered when evaluating PMT complications $[6-8,12]$. A previous study demonstrated upwards of $60 \%$ of patients who developed post-thrombectomy pancreatitis had chronic renal failure [7], contrasted with our patient who did not have pre-operative renal insufficiency. However, a limitation of this study was a total of four patients [7]. Current recommendations for intravascular catheter-based lysis consider limiting run time to 4 min in flowing vessels and 8 min within a thrombus $[6,11,14]$ may be difficult to adhere to when considering the significance and location of clot burden. Intraoperative fluid loss should be monitored carefully to gauge blood loss and potential erythrocyte damage, and in some cases, more than $50 \%$ of the effluent collected is composed of the patient's blood [15]. This may raise concern for excess circulating erythrocyte breakdown products and possible epithelial damage to distant organs.

If the cause of AP and AKI is known, removing the offending agent can prevent disease progression or future occurrences. AP is managed conservatively primarily with symptomatic control using aggressive fluid resuscitation and pain management [16]. AKI treatment depends on its etiology; however, refraining from nephrotoxic medication is essential [17]. In our patient, the use of unfractionated heparin was preferred over low molecular weight heparin, as well as cessation of non-steroidal anti-inflammatory drugs to prevent further renal impairment. In the event that TOS decompressive surgery is indicated, current recommendations endorse intervention during the same hospital stay $[4,5]$. This has been shown to reduce the period of anticoagulation, with patients returning to normal activity in a shorter time frame, and a decreased risk for recurrent thrombosis [4].

\section{Conclusion}

The exact pathophysiology of post-thrombectomy AP remains unknown; however, the possibilities include extensive hemolysis and epithelial damage from hemoglobin in addition to the possible systemic intravascular damage from FVL and intraoperative propofol administration. Further discussion and research into dosing and timing of tPA, as well as potentially limiting tPA administration to intraoperative or intensive care unit lysis may be useful in lowering the incidence of distant organ damage post-thrombectomy.

\section{Learning points}

AP is an uncommon sequela of PMT. Neutrophil recruitment from hemoglobin and hemoprotein breakdown from venous thrombi may be a contributing factor to acute pancreatic injury. The dosage and frequency of intraoperative propofol may factor into susceptibility to postoperative pancreatitis.

\section{Acknowledgments}

None to declare. 


\section{Financial Disclosure}

None to declare.

\section{Conflict of Interest}

None to declare.

\section{Informed Consent}

Informed consent was obtained from the patient regarding the publication of this case report.

\section{Author Contributions}

VM and SD wrote the article. SD and JD participated in the patient's care. JD supervised the article writing. All authors read and approved the manuscript, and ensure that this is the case.

\section{Data Availability}

The data supporting the findings of this study are available from the corresponding author upon reasonable request.

\section{Abbreviations}

PSS: Paget-Schroetter syndrome; PMT: percutaneous mechanical thrombectomy; AKI: acute kidney injury; AP: acute pancreatitis

\section{References}

1. Alla VM, Natarajan N, Kaushik M, Warrier R, Nair CK. Paget-schroetter syndrome: review of pathogenesis and treatment of effort thrombosis. West J Emerg Med. 2010;11(4):358-362.

2. Vemuri C, Salehi P, Benarroch-Gampel J, McLaughlin LN, Thompson RW. Diagnosis and treatment of effortinduced thrombosis of the axillary subclavian vein due to venous thoracic outlet syndrome. J Vasc Surg Venous Lymphat Disord. 2016;4(4):485-500.

3. Melby SJ, Vedantham S, Narra VR, Paletta GA, Jr., KhooSummers L, Driskill M, Thompson RW. Comprehensive surgical management of the competitive athlete with effort thrombosis of the subclavian vein (Paget-Schroetter syndrome). J Vasc Surg. 2008;47(4):809-820; discussion 821.
4. Schneider DB, Dimuzio PJ, Martin ND, Gordon RL, Wilson MW, Laberge JM, Kerlan RK, et al. Combination treatment of venous thoracic outlet syndrome: open surgical decompression and intraoperative angioplasty. J Vasc Surg. 2004;40(4):599-603.

5. Angle N, Gelabert HA, Farooq MM, Ahn SS, Caswell DR, Freischlag JA, Machleder HI. Safety and efficacy of early surgical decompression of the thoracic outlet for Paget-Schroetter syndrome. Ann Vasc Surg. 2001;15(1):3742.

6. Hershberger RC, Bornak A, Aulivola B, Mannava K. Acute pancreatitis after percutaneous mechanical thrombectomy: case report and review of the literature. Cardiovasc Intervent Radiol. 2011;34(Suppl 2):S25-30.

7. Lebow M, Cassada D, Grandas O, Stevens S, Goldman M, Freeman M. Acute pancreatitis as a complication of percutaneous mechanical thrombectomy. J Vasc Surg. 2007;46(2):366-368.

8. Piercy KT, Ayerdi J, Geary RL, Hansen KJ, Edwards MS. Acute pancreatitis: A complication associated with rheolytic mechanical thrombectomy of deep venous thrombosis. J Vasc Surg. 2006;44(5):1110-1113.

9. Kasirajan K, Gray B, Beavers FP, Clair DG, Greenberg $\mathrm{R}$, Mascha E, Ouriel K. Rheolytic thrombectomy in the management of acute and subacute limb-threatening ischemia. J Vasc Interv Radiol. 2001;12(4):413-421.

10. Perez-Pujol S, Aras O, Escolar G. Factor v leiden and inflammation. Thrombosis. 2012;2012:594986.

11. Likes K, Rochlin D, Nazarian SM, Streiff MB, Freischlag JA. Females with subclavian vein thrombosis may have an increased risk of hypercoagulability. JAMA Surg. 2013;148(1):44-49.

12. Druml W, Laggner AN, Lenz K, Grimm G, Schneeweiss B. Pancreatitis in acute hemolysis. Ann Hematol. 1991;63(1):39-41.

13. Haffar S, Kaur RJ, Garg SK, Hyder JA, Murad MH, Abu Dayyeh BK, Bazerbachi F. Acute pancreatitis associated with intravenous administration of propofol: evaluation of causality in a systematic review of the literature. Gastroenterol Rep (Oxf). 2019;7(1):13-23.

14. Vijaysadan V, Zimmerman AM, Pajaro RE. Paget-Schroetter syndrome in the young and active. J Am Board Fam Pract. 2005;18(4):314-319.

15. Muller-Hulsbeck S, Bathe M, Grimm J, Heller M. Enhancement of in vitro effectiveness for hydrodynamic thrombectomy devices. Simultaneous high-pressure rt-PA application. Invest Radiol. 1999;34(8):536-542.

16. Leppaniemi A, Tolonen M, Tarasconi A, Segovia-Lohse H, Gamberini E, Kirkpatrick AW, Ball CG, et al. 2019 WSES guidelines for the management of severe acute pancreatitis. World J Emerg Surg. 2019;14:27.

17. Moore PK, Hsu RK, Liu KD. Management of acute kidney injury: core curriculum 2018. Am J Kidney Dis. 2018;72(1):136-148. 